\title{
Estratégias de gerenciamento em listas de espera cirúrgicas: revisão integrativa
}

\author{
Management strategies on surgical waiting lists: integrative review
}

Estrategias de manejo en listas de espera quirúrgicas: revisión integradora

\author{
Rosa Ladi Lisbôa ${ }^{1 *}$, Camila Gabrielle da Silva Rodrigues ${ }^{1}$, Cristiane Vargas Bittencourt ${ }^{1}$, Adriana \\ Aparecida Paz ${ }^{1}$.
}

\section{RESUMO}

Objetivo: Identificar as estratégias utilizadas no gerenciamento de listas de espera de pacientes cirúrgicos eletivos. Métodos: Revisão integrativa realizada em quatro bases de dados, em janeiro de 2021. Utilizou-se a estratégia PICo para elaboração da questão de pesquisa. Os artigos foram gerenciados nos softwares Endnote X5 e Rayyan para a exclusão de documentos duplicados e cegamento dos avaliadores. Resultados: Obteve-se nove artigos que observaram a prevalência de pesquisas caso controle e estudos quantitativos (sete/77,77\%) classificados com nível de evidência quatro (moderado). Evidenciou-se a utilização de estratégias de gerenciamento para aproveitamento da equipe cirúrgica, centralização das listas de espera, colaboração de centros cirúrgicos de diferentes hospitais, status de priorização e tempo de espera do paciente, uso de software de computador e do método lean healthcare. Considerações finais: Este estudo pode contribuir para ampliar o conhecimento neste temário ainda pouco explorado no Brasil ao evidenciar a necessidade de compartilhamento de experiências exitosas para o aprimoramento do gerenciamento de listas de espera. As estratégias mostraram-se eficazes; entretanto, deve-se aprimorar algumas estratégias para que possam ser replicadas, respeitando as características específicas dos serviços de saúde no Brasil.

Palavras-chave: Administração de serviços de saúde, Listas de espera, Tecnologia aplicada, Sistema único de saúde, Enfermagem.

\begin{abstract}
Objective: To identify the strategies used to manage waiting lists for elective surgical patients. Methods: Integrative review carried out in four databases, in January 2021. The PICo strategy was used to elaborate the research question. The articles were managed in Endnote X5 and Rayyan software to exclude duplicate documents and blind the reviewers. Results: Nine articles were obtained that observed the prevalence of case-control research and quantitative studies (seven/77.77\%) classified with evidence level four (moderate). The use of management strategies to take advantage of the surgical team, centralization of waiting lists, collaboration of operating rooms from different hospitals, prioritization status and patient waiting time, use of computer software and the lean healthcare method was evidenced. Final considerations: This study can contribute to expand knowledge in this theme still little explored in Brazil by evidencing the need to share successful experiences for the improvement of the management of waiting lists. The strategies proved to be effective; however, some strategies must be improved so that they can be replicated, respecting the specific characteristics of health services in Brazil.
\end{abstract}

Keywords: Health services administration, Waiting lists, Technology applied, Unified health system, Nursing.

\section{RESUMEN}

Objetivo: Identificar las estrategias utilizadas para la gestión de listas de espera de pacientes quirúrgicos electivos. Métodos: Revisión integrativa realizada en cuatro bases de datos, en enero de 2021. Para la elaboración de la pregunta de investigación se utilizó la estrategia PICo. Los artículos se gestionaron en Endnote X5 y el software Rayyan para excluir documentos duplicados y cegar a los revisores. Resultados: Se obtuvieron nueve artículos que observaron la prevalencia de investigación de casos y controles y estudios cuantitativos (siete / 77,77\%) clasificados con nivel de evidencia cuatro (moderada). Se evidenció el uso de estrategias de gestión para el aprovechamiento del equipo quirúrgico, centralización de listas de espera, colaboración de centros quirúrgicos de diferentes hospitales, estado de priorización y tiempo de espera del paciente, uso de software informático y el método lean healthcare. Consideraciones finales: Este estudio puede contribuir para ampliar el conocimiento en este tema aún poco explorado en Brasil al evidenciar la necesidad de compartir experiencias exitosas para la mejora

\footnotetext{
${ }^{1}$ Universidade Federal de Ciências da Saúde de Porto Alegre (UFCSPA), Porto Alegre - RS.

*E-mail: rosaladilisboa@gmail.com
}

SUBMETIDO EM: 1/2022

ACEITO EM: 1/2022

PUBLICADO EM: 2/2022 
de la gestión de las listas de espera. Las estrategias demostraron ser efectivas; sin embargo, es necesario mejorar algunas estrategias para que puedan ser replicadas, respetando las características específicas de los servicios de salud en Brasil.

Palabras clave: Administración de los servicios de salud, Listas de espera, Tecnología aplicada, Sistema único de salud, Enfermería.

\section{INTRODUÇÃO}

As listas de espera para cirurgias eletivas são consideradas uma das principais preocupações das políticas de saúde na maioria dos países do mundo. A razão mais comum para explicar esse fenômeno é que a demanda excede a oferta (JULIO C, et al., 2016). Este fato que torna o acesso à atenção especializada mais complicado, seja por conta de especificidades do sistema, como maior cobertura da atenção básica ou a falta de recursos e de serviços, ou do perfil dos usuários, cuja expectativa de vida aumentou assim como apresentam doenças crônicas (FARIAS CML, et al., 2019).

A otimização do gerenciamento de listas de espera de pacientes cirúrgicos eletivos é de extrema importância para o funcionamento do sistema de saúde visto que demoras na realização dessas cirurgias potencializam uma série de problemas para os pacientes tais como piora/deterioração nas condições de saúde, prolongamento do sofrimento físico e psíquico, perda de utilidade/função corporal e evolução para o óbito (SICILIANI L e HURST J, 2005).

O longo período de espera de um paciente em uma lista pode repercutir no paciente e em sua família, médico, hospital, sistema de saúde e toda a sociedade dependente deste sistema. Para o paciente, essa espera, além de ser angustiante por não tratar do seu problema de imediato, pode causar complicações como o agravamento do seu estado inicial e até mesmo a morte. Por conseguinte, o adiamento do procedimento inicial torna o quadro do paciente mais complexo para os médicos e equipes, gerando gastos maiores para o hospital com procedimentos e no acompanhamento pós-cirúrgico dos pacientes (SENNA SBB, et al., 2020).

Além disso, ainda do ponto de vista econômico, pacientes que têm seu quadro clínico agravado por conta de uma longa espera pelo procedimento tendem a permanecer mais tempo hospitalizados, utilizando, portanto, mais recursos e ampliando a exposição aos riscos de eventos adversos como as infecções hospitalares. Nessas condições, forma-se um ciclo vicioso na assistência repleto de prejuízos imediatos para o paciente e a sociedade (LISBÔA RL, et al., 2022).

O tempo médio de espera para uma cirurgia eletiva no mundo varia de pelo menos três meses, podendo chegar a anos. No Brasil, em 2017, considerando 16 estados, havia mais de 800 mil pacientes na lista de espera de procedimentos eletivos pelo Sistema Único de Saúde (SUS), sendo que o paciente mais antigo estava aguardando há 12 anos (GIAMBERNARDINO FD, 2019). Estes números revelam a lentidão do sistema em prestar os serviços de atenção à saúde demandados pela população, o que pode afetar não somente 0 prognóstico, como também a qualidade de vida dos pacientes acometidos por doenças cujos sintomas são graves ou estigmatizantes. Assim, além do já mencionado aumento nos gastos com saúde, a eficácia da clínica é comprometida por conta de tempos de espera longos (FARIAS CML, et al., 2019).

Destaca-se que a pandemia ocasionada pela Covid-19 causou impacto significativo na prestação de serviços de saúde, incluindo consultas e volumes operativos, gerando desafios significativos para o sistema de saúde superar o acúmulo não só de consultas, mas também de cirurgias e nos tempos de espera cirúrgica (SAGGAF M e ANASTAKI DJ, 2021).

Criado pela Constituição Federal de 1988, o SUS vem buscando continuamente a sua consolidação, por meio de políticas, projetos e ações, de forma a qualificar-se e alcançar cada vez mais seus princípios, tais como acessibilidade, integralidade, equidade, eficiência, entre outros. Contudo, em termos legais, existe uma carência de determinações governamentais a respeito da gestão e da transparência das listas de espera, apesar da existência do Projeto de Lei Federal no 393/2015, em tramitação, que dispõe sobre a obrigatoriedade de publicação e a atualização sistemática no sítio eletrônico do hospital da lista de espera dos pacientes para cirurgias eletivas pelo SUS (AGUIAR LOF e LIRA ACO, 2018).

Juntamente com a falta de orientações legais específicas, há o problema de escassez de produção literária sobre a questão. Embora o tema das listas e dos tempos de espera para cirurgias eletivas venha sendo 
relatado internacionalmente como alvo de políticas de saúde desde a década de 90 , no Brasil, não foi encontrado um arsenal literário consistente. Dessa forma, não foi encontrada literatura deste temário que contemple uma padronização de estratégias utilizadas no gerenciamento de listas de espera pelos núcleos de regulação dos serviços de saúde (LIPPI MC, et al., 2018).

Este estudo é resultado da vivência laboral em um Núcleo Interno de Regulação (NIR) do maior hospital do Sul do Brasil, cujo atendimento é feito exclusivamente pelo SUS. Neste NIR a mediação do processo de regulação das listas de espera cirúrgicas é realizada pelo enfermeiro, que fortalece a análise de indicadores e a formulação de estratégias para a tomada de decisões em nível de gestão (LISBÔA RL, et al., 2022). A gestão de leitos nas instituições hospitalares é uma área de atuação em que o enfermeiro possui autonomia, realiza tomada de decisões e exerce o gerenciamento e liderança (LISBÔA RL, et al., 2021).

A gestão de listas de espera é uma recente área de atuação do enfermeiro; trata-se de um trabalho desafiador, que não possui tantas referências para embasar a realização desta tarefa (LISBÔA RL, et al., 2022). Dessa forma, conhecer, identificar e analisar as estratégias de gerenciamento em listas de espera é fundamental para atingir os objetivos e metas da regulação de leitos hospitalares. Estratégias adequadas garantem bons resultados, e sua análise pode servir como inspiração para a implantação em outros serviços, ou como uma experiência a não ser replicada. Neste artigo, por meio desta revisão integrativa, objetivou-se identificar as estratégias utilizadas no gerenciamento de listas de espera de pacientes cirúrgicos eletivos.

\section{MÉTODOS}

Trata-se de um estudo de abordagem qualitativa e de síntese da produção científica realizada graças à utilização do método de Revisão Integrativa de Literatura (RIL). Este método apresenta no seu percurso seis etapas distintas e complementares que consistem na questão de pesquisa, a amostragem ou busca na literatura, categorização dos estudos, avaliação dos estudos selecionados, interpretação dos resultados e, por fim, a apresentação da revisão (MENDES KDS, et al., 2019).

A questão de pesquisa foi "Quais são as estratégias utilizadas no gerenciamento da lista de espera de pacientes cirúrgicos?". Utilizou-se a estratégia PICo, sendo que nossa população $(\mathrm{P})$ foi composta pelos pacientes cirúrgicos em lista de espera; o fenômeno de interesse a ser considerado (I) trata das estratégias de gerenciamento de listas de espera cirúrgicas, e o contexto (Co) remetendo à diminuição do tempo de espera para a cirurgia e à otimização dos leitos hospitalares cirúrgicos (BRASIL, 2014).

Para a busca nas bases de dados, foram utilizados os descritores combinados por intermédio dos operadores booleanos AND e OR. Sendo assim, a estratégia elaborada para a busca nas bases de dados foi "Elective Surgical Procedures" OR "Health Services Administration" AND "Waiting Lists" OR "Technology Applied to Waiting Lists".

As bases de dados pesquisadas foram National Library of Medicine (PubMed), Biblioteca Virtual em Saúde (BVS), Web of Science (WoS) e a SCOPUS. Definiu-se a pesquisa nestas bases de dados por elas atingirem maior amplitude do acervo literário científico. Foram incluídos artigos científicos nos idiomas português, inglês ou espanhol e disponíveis em texto completo.

Considerou-se como critérios de exclusão: não responder à questão de pesquisa; textos no formato editoriais, monografias, dissertações, teses, revisão integrativa ou bibliográfica, resumos simples ou expandidos; e estudos com falhas metodológicas graves identificados na análise crítica da leitura na íntegra.

A coleta dos dados ocorreu nas bases de dados em janeiro de 2021, referente às publicações ocorridas no período de 2016 a 2020. Foram utilizados o Endnote X5, versão desktop, para a exclusão de artigos duplicados, e o aplicativo Rayyan Qatar Computing Research Institute (QCRI) (http://rayyan.qcri.org/) para a avaliação cegada (OUZZANI M, et al., 2016; SOUZA LB, et al., 2021). Destaca-se que os softwares otimizaram o gerenciamento das etapas de leituras textuais. As avaliadoras realizaram a análise de forma organizada, com possibilidade de registrar a justificativa de cada documento, contribuindo para a expressão dos resultados (REIGOTA SM, et al., 2019).

A avaliação dos artigos adotada foi a de revisão por pares (peer review), ocorrendo a avaliação de uma terceira pessoa nos documentos que tiveram caso de discordância entre os pares. O corpus de análise é 
constituído por nove artigos científicos que discorrem sobre estratégias de gerenciamento em listas de espera cirúrgicas. A Figura 1 apresenta as etapas do processo de seleção, que seguiu os princípios do modelo/diagrama Preferred Reporting Items for Systematic Reviews and Meta-Analyses (PRISMA), e apresenta o fluxo desde a estratégia de seleção dos artigos até a obtenção da amostra final (MOHER D, et al., 2009; PAGE MJ, et al., 2021). O fluxograma das etapas que compõem este estudo pode ser observado na Figura 1.

Figura 1 - Fluxograma das etapas de seleção das produções para compor o corpus final $(n=9)$.

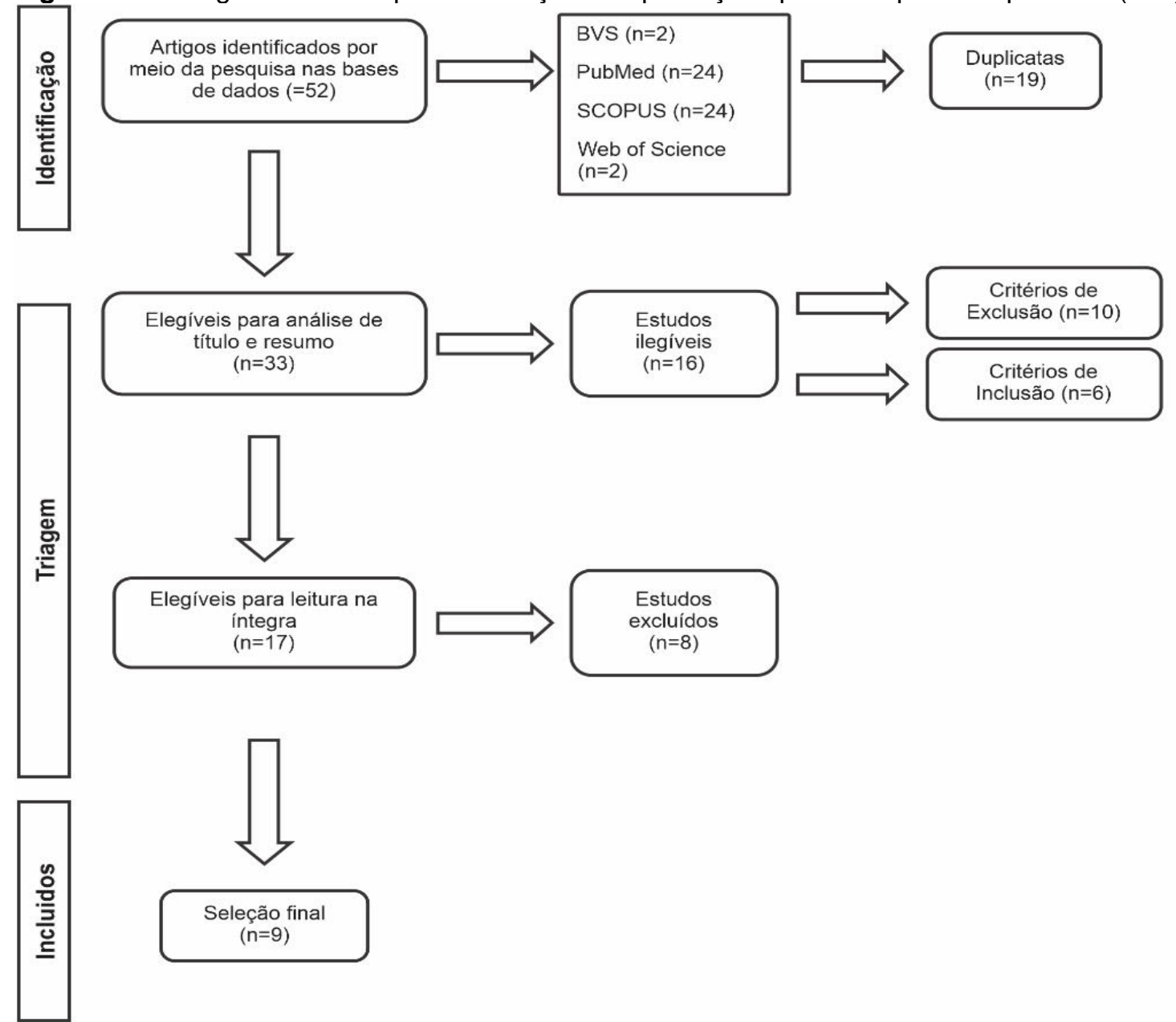

Fonte: Lisbôa RL, et al., 2022.

O nível de evidência foi classificado conforme os instrumentos de avaliação propostos pela Joanna Briggs Institute (JBI). A classificação define o nível em: revisões sistemáticas ou metanálises de ensaios clínicos randomizados relevantes (N1); ensaios clínicos randomizados (N2); ensaios clínicos controlados sem randomização (N3); casos controle e estudo de coorte (N4); revisões sistemáticas de estudos qualitativos ou descritivos (N5); evidência de um único estudo descritivo ou qualitativo (N6); e parecer de autoridades e/ou relatórios de comitês de especialistas (N7) (MELNYK BM e FINEOUT-OVERHOLT E, 2011; JOST MT, et al., 2018). Esta hierarquia classifica os níveis 1 a 2 como fortes, 3 a 5 como moderados e 6 a 7 como fracos.

Utiliza-se a análise descritiva na apresentação dos resultados a seguir. Ressalta-se que a expressão de ideias e fatos descritos nos textos analisados foi preservada, respeitando a Lei de Direitos Autorais (BRASIL, 2013).

\section{RESULTADOS}

A seleção final de nove artigos que integram esta pesquisa evidencia a lacuna de conhecimento neste temário. O período de publicação que teve a maior prevalência ocorreu em 2017, no quantitativo de três 
(33,33\%), seguido de 2016 e 2019 com dois (22,22\%) artigos em cada ano, e, nos anos de 2018 e 2020 , apenas um $(11,11 \%)$ artigo em cada ano. Os artigos selecionados contemplam o cenário mundial, sendo quatro oriundos da América do Norte, Canadá e Estados Unidos com dois $(22,22 \%)$ cada um. Os demais cinco estudos têm a procedência única dos países: Inglaterra, Noruega, Itália, Índia e Chile.

O tema caracteriza-se por ser abrangente e multidisciplinar. A maioria dos artigos (seis/66,66\%) foi publicada em revistas com enfoque na área de Organização e Gestão em Saúde, com caráter interdisciplinar que visam à disseminação de políticas de saúde e pesquisa gerencial. Entretanto, três (33,33\%) artigos foram divulgados em periódicos da área médica. O idioma predominante dos artigos (oito/88,88\%) foi o inglês, existindo apenas um em espanhol. No Quadro 1, são apresentados os estudos que buscaram relatar e avaliar sobre estratégias de gerenciamento em listas de espera cirúrgicas.

Quadro 1 - Autor, ano, título, objeto de estudo, Nível de Evidência (NE) dos artigos selecionados.

\begin{tabular}{|c|c|c|c|c|}
\hline $\begin{array}{l}\text { Autores } \\
\text { (Ano) }\end{array}$ & Título & Objetivo do estudo & Delineamento & NE \\
\hline $\begin{array}{l}\text { Clarke J, et } \\
\text { al. (2020) }\end{array}$ & $\begin{array}{l}\text { New geographic model of care } \\
\text { to manage the post-COVID-19 } \\
\text { elective surgery aftershock in } \\
\text { England: a retrospective } \\
\text { observational study }\end{array}$ & $\begin{array}{l}\text { Aplicar a detecção de comunidade } \\
\text { multiescala Markov (MMCD), uma estrutura } \\
\text { de agrupamento baseada em gráficos não } \\
\text { supervisionados para identificar novos } \\
\text { modelos de atendimento cirúrgico. }\end{array}$ & Caso controle & N4 \\
\hline $\begin{array}{l}\text { Gangstoe } \\
\text { JJ, et al. } \\
(2016)\end{array}$ & $\begin{array}{l}\text { Norwegian priority setting in } \\
\text { practice - an analysis of waiting } \\
\text { time patterns across medical } \\
\text { disciplines }\end{array}$ & $\begin{array}{l}\text { Estudar a variação no status de prioridade e } \\
\text { no tempo de espera de pacientes em } \\
\text { diferentes especialidades médicas. }\end{array}$ & Caso controle & N4 \\
\hline $\begin{array}{l}\text { Laberge M, } \\
\text { et al. (2019) }\end{array}$ & $\begin{array}{l}\text { Clinical pathway efficiency for } \\
\text { elective joint replacement } \\
\text { surgeries: a case study }\end{array}$ & $\begin{array}{l}\text { Definir uma via clínica para a cirurgia de } \\
\text { substituição total da articulação (TJR), } \\
\text { estimar o efeito dos atrasos entre as etapas } \\
\text { do processo ate a realização do } \\
\text { procedimento cirúrgico, identificar os fatores } \\
\text { que contribuem para operações mais } \\
\text { eficientes e os desafios para sua } \\
\text { implementação. }\end{array}$ & Caso controle & N4 \\
\hline $\begin{array}{l}\text { Lungu DA, } \\
\text { et al. (2019) }\end{array}$ & $\begin{array}{l}\text { Decision making tools for } \\
\text { managing waiting times and } \\
\text { treatment rates in elective } \\
\text { surgery }(2019)\end{array}$ & $\begin{array}{l}\text { Analisar a relação entre tempos de espera e } \\
\text { taxas de tratamento para nove } \\
\text { procedimentos cirúrgicos eletivos de alto } \\
\text { volume, a fim de apoiar a tomada de decisão } \\
\text { quanto à disponibilidade desses serviços } \\
\text { para os cidadãos. }\end{array}$ & Caso controle & N4 \\
\hline $\begin{array}{l}\text { Pomey MP, } \\
\text { et al. (2017) }\end{array}$ & $\begin{array}{l}\text { Wait time management } \\
\text { strategies for } \begin{array}{r}\text { total joint } \\
\text { replacement }\end{array} \\
\text { sustainability and unintended } \\
\text { consequences }\end{array}$ & $\begin{array}{l}\text { Avaliar implementação de Estratégias de } \\
\text { gerenciamento de lista. }\end{array}$ & Caso controle & N4 \\
\hline $\begin{array}{l}\text { Valsangkar } \\
\text { NP, et al. } \\
\text { (2017) }\end{array}$ & $\begin{array}{l}\text { Effect of Lean Processes on } \\
\text { Surgical Wait Times and } \\
\text { Efficiency in a Tertiary Care } \\
\text { Veterans Affairs Medical } \\
\text { Center }\end{array}$ & $\begin{array}{l}\text { Identificar se os processos enxutos podem } \\
\text { ser usados para melhorar os tempos de } \\
\text { espera para procedimentos cirúrgicos em } \\
\text { hospitais de veteranos de guerra. }\end{array}$ & Caso controle & N4 \\
\hline $\begin{array}{l}\text { Ray S, } \\
\text { Kirtania J. } \\
(2017)\end{array}$ & 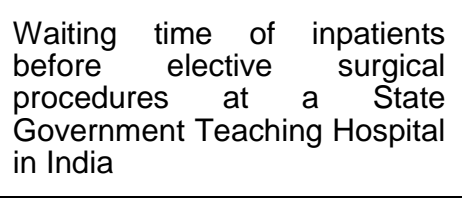 & $\begin{array}{l}\text { Mensurar o tempo de espera do paciente } \\
\text { internado, identificar os fatores que } \\
\text { interferem neste tempo e recomendar formas } \\
\text { de reduzi-lo antes do procedimento cirúrgico } \\
\text { eletivo, em um hospital universitário do } \\
\text { governo estadual na Índia. }\end{array}$ & Caso controle & N4 \\
\hline $\begin{array}{l}\text { Julio C, et } \\
\text { al. (2016) }\end{array}$ & $\begin{array}{l}\text { Waiting lists management } \\
\text { model based on timeliness and } \\
\text { justice }\end{array}$ & $\begin{array}{l}\text { Propor um modelo de gestão de lista de } \\
\text { espera que avance na conciliação entre } \\
\text { oferta e demanda no Chile, por meio de uma } \\
\text { caracterização clínica da lista e de um } \\
\text { processo de tomada de decisão que garanta } \\
\text { oportunidade e justiça no atendimento. }\end{array}$ & $\begin{array}{l}\text { Transversal } \\
\text { /Descritivo }\end{array}$ & N6 \\
\hline $\begin{array}{l}\text { Logvinov II, } \\
\text { et al. (2018) }\end{array}$ & $\begin{array}{l}\text { Patient survey of referral from } \\
\text { one surgeon to another to } \\
\text { reduce maximum waiting time } \\
\text { for elective surgery and hours } \\
\text { of overutilized operating room } \\
\text { time }\end{array}$ & $\begin{array}{l}\text { Identificar a opinião do paciente sobre a troca } \\
\text { de cirurgião, nos casos em que a troca } \\
\text { pudesse diminuir o tempo de espera para } \\
\text { realização do procedimento. }\end{array}$ & $\begin{array}{l}\text { Transversal } \\
\text { /Descritivo }\end{array}$ & N6 \\
\hline
\end{tabular}

Fonte: Lisbôa RL, et al., 2022. 
Com relação ao tipo de pesquisa, observou-se a prevalência de pesquisas caso controle de abordagem quantitativa (sete/77,77\%), conforme a classificação do nível de evidência pela JBI (MELNYK BM e FINEOUTOVERHOLT E, 2011; JOST MT, et al., 2018). Os estudos desta revisão foram predominantemente de nível moderado.

No Quadro 2, observam-se a síntese dos principais resultados, a conclusão e as estratégias de gerenciamento da lista de espera encontrados.

Quadro 2 - Resultados, conclusão, estratégia de gerenciamento da lista de espera dos estudos selecionados.

\begin{tabular}{|c|c|c|c|}
\hline $\begin{array}{c}\text { Autores } \\
\text { (Ano) }\end{array}$ & Resultados & Conclusão & Estratégia \\
\hline $\begin{array}{l}\text { Clarke J, et al. } \\
\quad(2020)\end{array}$ & $\begin{array}{l}\text { A média do deslocamento de pacientes } \\
\text { para realização dos procedimentos ao } \\
\text { hospital foi de } 11,3 \text { quilômetros. }\end{array}$ & $\begin{array}{l}\text { Listas de espera agrupadas para } \\
\text { procedimentos eletivos de baixo risco e } \\
\text { pacientes em redes de comunidades } \\
\text { cirúrgicas expandidas e integradas têm o } \\
\text { potencial de aumentar a eficiência. }\end{array}$ & $\begin{array}{l}\text { Centralização do } \\
\text { gerenciamento das listas } \\
\text { de espera cirúrgicas. } \\
\text { Colaboração entre centros } \\
\text { cirúrgicos de diferentes } \\
\text { hospitais na realização de } \\
\text { procedimentos. }\end{array}$ \\
\hline $\begin{array}{l}\text { Gangstoe JJ, } \\
\text { et al. (2016) }\end{array}$ & $\begin{array}{l}\text { Encontrou-se uma variação considerável } \\
\text { entre as especialidades médicas quanto } \\
\text { à proporção de pacientes prioritários e } \\
\text { seus tempos máximos de espera. O grau } \\
\text { de diferenciação dos tempos máximos de } \\
\text { espera também variou de acordo com a } \\
\text { especialidade médica. }\end{array}$ & $\begin{array}{l}\text { A falta de correspondência clara do } \\
\text { tempo real de espera e do status de } \\
\text { prioridade pode implicar que a urgência, } \\
\text { descrita na carta de encaminhamento, e } \\
\text { a gravidade da doença, de acordo com } \\
\text { as diretrizes, sejam duas entidades } \\
\text { distintas. }\end{array}$ & $\begin{array}{l}\text { Gerenciamento de lista de } \\
\text { espera baseado no status } \\
\text { de priorização do paciente } \\
\text { e tempo de espera. }\end{array}$ \\
\hline $\begin{array}{l}\text { Laberge M, et } \\
\text { al. (2019) }\end{array}$ & $\begin{array}{l}\text { Observou grandes variações nos atrasos } \\
\text { entre as sete etapas identificadas. O } \\
\text { atraso entre a Etapa } 1 \text { e a Etapa } 2 \text { foi a } \\
\text { única variável significativa na previsão do } \\
\text { tempo total de espera até a cirurgia. }\end{array}$ & $\begin{array}{l}\text { A abordagem permitiu um melhor } \\
\text { entendimento dos processos, da cultura } \\
\text { organizacional e como estes podem } \\
\text { afetar uns aos outros. Evidenciou-se que } \\
\text { a cultura e as práticas organizacionais } \\
\text { são mais difíceis de influenciar. }\end{array}$ & $\begin{array}{l}\text { Método lean healthcare. } \\
\text { Mapeamento } \\
\text { identificação de falhas no } \\
\text { processo de cirurgia. }\end{array}$ \\
\hline $\begin{array}{l}\text { Lungu DA, et } \\
\text { al. (2019) }\end{array}$ & $\begin{array}{l}\text { A análise realizada para as nove } \\
\text { intervenções mostra que os } 34 \text { distritos } \\
\text { estão em posições variáveis na matriz } \\
\text { tempo de espera e taxa de tratamento, } \\
\text { sugerindo que não existe uma relação } \\
\text { direta entre taxas e tempos de espera. }\end{array}$ & $\begin{array}{l}\text { Os formuladores de políticas e gestores } \\
\text { de saúde seguem uma estratégia de } \\
\text { duas etapas: identificam o tipo de } \\
\text { contexto e, em seguida, analisam o } \\
\text { impacto na produtividade e } \\
\text { disponibilidade dos recursos, } \\
\text { preferências dos pacientes e adequação } \\
\text { do cuidado. }\end{array}$ & $\begin{array}{l}\text { Participação } r \text { e } \\
\text { engajamento da equipe } \\
\text { médica. Decisão dos } \\
\text { pacientes sobre onde } \\
\text { realizar o procedimento. }\end{array}$ \\
\hline $\begin{array}{l}\text { Pomey MP, et } \\
\text { al. (2017) }\end{array}$ & $\begin{array}{l}\text { As organizações de sucesso tinham } \\
\text { características específicas: } \\
\text { gerenciamento total do processo de } \\
\text { atendimento; 2) forte liderança clínica; 3) } \\
\text { comitês dedicados para coordenar e } \\
\text { sustentar a estratégia; 4) cultura baseada } \\
\text { na confiança e na inovação. }\end{array}$ & $\begin{array}{l}\text { Para serem sustentáveis, as estratégias } \\
\text { de gerenciamento do tempo de espera } \\
\text { precisam gerar maiores sinergias entre a } \\
\text { estratégia de nível contextual (provincial } \\
\text { ou regional) e os objetivos e restrições } \\
\text { organizacionais. }\end{array}$ & $\begin{array}{lr}\text { Centralização } & \text { do } \\
\text { gerenciamento de listas de } \\
\text { espera. } \quad \text { Equipes } \\
\text { engajadas, atuando com } \\
\text { liderança. Criação de } \\
\text { comitês para coordenar } \\
\text { estratégias. }\end{array}$ \\
\hline $\begin{array}{l}\text { Valsangkar } \\
\text { NP, et al. } \\
(2017)\end{array}$ & $\begin{array}{l}\text { Após as implementações de projetos de } \\
\text { workshop de melhoria rápida de } \\
\text { processo, o tempo médio de espera para } \\
\text { procedimentos cirúrgicos gerais eletivos } \\
\text { diminuiu de } 33,4( \pm 8,3) \text { dias no ano fiscal } \\
\text { de } 2012 \text { para } 26( \pm 9,5) \text { em } 2013(\mathrm{P}=02) \text {. } \\
\text { No ano de } 2014 \text {, os tempos de espera } \\
\text { médios foram metade do valor do ano } \\
\text { fiscal anterior em } 12( \pm 2,1) \text { dias }(\mathrm{P}=07) \text {. }\end{array}$ & $\begin{array}{l}\text { A melhoria na experiência geral do } \\
\text { paciente cirúrgico pode resultar da } \\
\text { colaboração multidisciplinar entre a } \\
\text { equipe de redesenho de sistemas, } \\
\text { médicos e equipe cirúrgica para reduzir } \\
\text { ineficiências sistêmicas. }\end{array}$ & $\begin{array}{l}\text { Aplicação do método lean } \\
\text { healthcare para redução } \\
\text { do tempo de espera. }\end{array}$ \\
\hline $\begin{array}{l}\text { Ray S, } \\
\text { Kirtania J. } \\
(2017)\end{array}$ & $\begin{array}{l}\text { O tempo mediano de espera dos } \\
\text { pacientes internados antes da cirurgia } \\
\text { eletiva foi de } 12 \text { dias, sendo influenciado } \\
\text { significativamente }(p<0,05) \text { pela idade do } \\
\text { paciente, estado físico e financeiro. }\end{array}$ & $\begin{array}{l}\text { Foram identificadas medidas de controle } \\
\text { para reduzir o tempo de espera dos } \\
\text { pacientes internados antes de cirurgias } \\
\text { eletivas. }\end{array}$ & $\begin{array}{l}\text { Reduzir o tempo de espera } \\
\text { de internação para } \\
\text { contatar outros pacientes } \\
\text { eletivos. }\end{array}$ \\
\hline $\begin{array}{l}\text { Julio C, et al. } \\
\qquad(2016)\end{array}$ & $\begin{array}{l}\text { A implantação de um software de } \\
\text { computador para cadastrar pacientes, } \\
\text { priorizar e monitorar as listas de espera } \\
\text { evidenciou que a tempestividade não é } \\
\text { suficiente para gerenciar as listas de } \\
\text { espera para cirurgias eletivas, sendo } \\
\text { esta complementada com um indicador } \\
\text { de justiça. }\end{array}$ & $\begin{array}{l}\text { Os hospitais devem tentar equilibrar } \\
\text { justiça e pontualidade no atendimento e } \\
\text { priorizar iniciativas que aprimorem os } \\
\text { dois indicadores ao mesmo tempo. }\end{array}$ & $\begin{array}{l}\text { Utilização do software de } \\
\text { computador. }\end{array}$ \\
\hline $\begin{array}{l}\text { Logvinov II, et } \\
\text { al. (2018) }\end{array}$ & $\begin{array}{l}\text { Mais de } 50 \% \text { dos pacientes gostariam de } \\
\text { discutir a opção de realizar o } \\
\text { procedimento com outro cirurgião na } \\
\text { situação de reduzir o tempo de espera. }\end{array}$ & $\begin{array}{l}\text { O paciente deseja discutir com a equipe } \\
\text { cirúrgica a opção por outro cirurgião. A } \\
\text { decisão compartilhada resultaria em uma } \\
\text { redução considerável no tempo médio de } \\
\text { espera, tempo de sala de cirurgia } \\
\text { subutilizado, redução de horas extras ou } \\
\text { adicional noturno da equipe cirúrgica, } \\
\text { como de anestesistas e enfermagem. }\end{array}$ & $\begin{array}{l}\text { Aproveitamento da equipe } \\
\text { cirúrgica. }\end{array}$ \\
\hline
\end{tabular}

Fonte: Lisbôa RL, et al., 2022. 


\section{DISCUSSÃO}

A capacidade dos sistemas públicos de saúde é limitada em financiamento, especialistas e outros recursos. Uma maneira de lidar com esta situação é priorizar o atendimento de problemas de saúde que melhoram o resultado de saúde do sistema. Do lado da demanda, há evidências de que os custos associados à espera por uma intervenção cirúrgica estão relacionados a efeitos sociais, efeitos psicológicos, grau de incapacidade e probabilidade de deterioração do estado de saúde do paciente, sendo que este último risco pode ser reduzido se forem estabelecidos critérios para a priorização do atendimento aos pacientes (JULIO C, et al., 2016; LISBÔA RL, et al., 2022).

Prioridade representa a posição relativa de uma pessoa na lista de espera em um determinado momento. É uma visão do paciente em contexto, segundo a qual seu lugar na lista pode ser construído a partir da urgência de atender sua patologia e dos demais pacientes que aguardam. Assim, o nível de urgência é centrado em um paciente, ao passo que a prioridade é centrada em todos os pacientes (JULIO C, et al., 2016).

Para avançar nesse sentido, é proposto um modelo de gestão da lista de espera que inclua a justiça de forma complementar à oportunidade de cuidado. Um modelo definido desta forma permite avaliar a gestão de listas espera de uma perspectiva social, com base na equidade de acesso e resultados de saúde, mas sem perder de vista o olhar centrado no paciente. Os resultados dessa experiência mostram que os hospitais devem manter um equilíbrio entre esses indicadores e se concentrar no desenvolvimento de iniciativas que melhorem as oportunidades e a justiça simultaneamente (JULIO C, et al., 2016; CLARKE J, et al., 2020).

A estratégia de priorização dos pacientes no gerenciamento de listas de espera cirúrgicas constitui-se como uma importante medida que atende ao princípio doutrinário da equidade do SUS. Este princípio precisa ser incorporado no gerenciamento de procedimentos cirúrgicos para atender as necessidades do paciente. A busca pela equidade em saúde constitui-se em uma forma de garantir um direito social - a saúde (ALBRECHT CAM, et al., 2017; LOPES F, 2021).

O estudo realizado no Chile descreve a estratégia do uso de software no gerenciamento de listas. Esta aplicação evidenciou a necessidade de agregar outros indicadores que contemplem as diferenças e a realidade das condições clínico-cirúrgicas dos pacientes para acolher com justiça quem precisa do procedimento (JULIO C, et al., 2016; CLARKE J, et al., 2020).

Os sistemas eletrônicos podem facilitar a coleta, o armazenamento, a recuperação, o monitoramento e a disseminação de informações de forma ágil, segura e fidedigna de modo a contribuir na qualidade das funções gerenciais, operacionais e tomada de decisão (OLIVEIRA IS, et al., 2021).

Os estudos encontrados são internacionais, e a equidade é um dos princípios do sistema de saúde vigente no Brasil (LOPES F, 2021). Dessa maneira, torna-se evidente que esse critério precisa ser mantido e valorizado na regulação, pois as pessoas são e possuem necessidades diferentes entre si. Para ser justo e agir com humanização, torna-se imprescindível considerar a equidade como característica indispensável nos serviços de saúde. Portanto, ratifica-se a necessidade de os sistemas eletrônicos possuírem requisitos que contemplem a equidade no acesso dos pacientes (JULIO C, et al., 2016; LISBÔA RL, et al., 2022).

A necessidade de melhorias na qualidade dos serviços assim como no gerenciamento dos recursos demanda o uso de métodos e ferramentas para a área da saúde, como a produção enxuta, por exemplo REGIS TK, et al., 2018; SILVA TO, et al., 2019). Quanto ao método, após revisão em dois estudos, verificouse que o método lean healthcare é uma estratégia de gerenciamento utilizada em muitos países na área da saúde com vistas a beneficiar tanto os profissionais e gestores de saúde quanto os pacientes, pois objetiva uma melhor eficiência, satisfação e segurança dos processos referentes aos serviços prestados (SANTOS MC e BALSANELLI APB, 2021).

Este método é um sistema de gestão focado na eliminação de desperdícios para que se otimizem recursos e os resultados sejam favoráveis, contemplando a qualidade e segurança. O Lean se dá através de práticas que visam ao cumprimento de normas que agregam valor nas organizações de forma a conservar a boa imagem da instituição, melhorando o seu processo de trabalho (SILVA LID, et al., 2021).

A proposição de melhoria do processo, baseada nesta metodologia, tem como objetivos: eliminar etapas desnecessárias, reduzir o tempo de espera no atendimento, aprimorar e tornar mais eficiente os processos, 
melhorar a comunicação entre as equipes de saúde, pacientes e familiares, eliminar ou reduzir os desperdícios existentes no processo, seja na recepção, procedimento assistencial ou de organização. A metodologia Lean, nas instituições de saúde vem sendo utilizada com o propósito de melhorar a qualidade dos processos e serviços em diversos cenários da instituição hospitalar (SANTOS LM, et al., 2020).

Destaca-se, neste sentido, que um dos principais recursos do lean healthcare é o tempo dentro do qual é possível identificar os eventos que podem impedir ou atrasar o planejamento de cirurgias; assim, propõemse ações de controle para alcance de resultados positivos. Esse método não envolve um valor financeiro significativo, mas sua prática oportuniza avanços e melhorias no serviço, especialmente na organização das listas e na redução do tempo de espera dos pacientes eletivos (VALSANGKAR NP, et al., 2017).

A redução do tempo de internação dos pacientes também foi encontrada como uma estratégia, pois repercute diretamente na quantidade de pacientes eletivos para o procedimento cirúrgico. Todavia, quanto mais rápida e eficiente a hospitalização, maior o fluxo de rotatividade e resolutividade cirúrgica para os pacientes (LISBÔA RL, et al., 2022).

A estratégia de centralização das listas de espera para o adequado gerenciamento foi evidenciada como uma ferramenta importante para evitar a fragmentação que dificulta sobremaneira a interação e a consequente eficácia no atendimento aos pacientes cirúrgicos eletivos. Ressalta-se que a centralização permite aos gestores a visibilidade do panorama geral e da realidade institucional. Além disso, possibilita a integração dos diferentes serviços de saúde necessários para a realização do procedimento cirúrgico (POMEY MP, et al., 2017; CLARKE J, et al., 2020).

Destaca-se como estratégia a colaboração de diferentes hospitais no atendimento ao paciente eletivo. Na Itália, o paciente pode ser atendido em qualquer hospital que possua condições para a realização do procedimento, não sendo necessário aguardar o agendamento pela equipe médica emitente do laudo de indicação cirúrgica, como no Brasil (LUNGU DA, et al., 2019).

Identificaram-se outras estratégias de gerenciamento das listas, como a possibilidade de o paciente escolher outro cirurgião que tenha maior disponibilidade de agenda na realização do procedimento, o que consequentemente reduz o tempo de espera, evidenciando que o aproveitamento da equipe médica é uma estratégia importante, assim como usufruir da organização estrutural e dos demais profissionais que atuam no centro cirúrgico (LUNGU DA, et al., 2019; LOGVINOV II, et al., 2018).

Como limitação deste estudo, elenca-se o número reduzido de publicações encontradas neste temário. Ao mesmo tempo, revelou-se uma lacuna de conhecimento na área, o que incentiva a investir em novos estudos.

\section{CONSIDERAÇÕES FINAIS}

Embora as estratégias identificadas mostrem-se eficazes nos cenários em conformidade com seus sistemas de saúde, evidenciou-se que não existe uma padronização de estratégias no gerenciamento de listas de espera cirúrgicas. Possivelmente, a diferença entre os sistemas de saúde no mundo contribui para a limitação da padronização de estratégias. Neste sentido, ratifica-se que as diferenças precisam de fato ser contempladas, levando-se em consideração as características da população e o contexto em que estão inseridas para que o serviço prestado possa ser mais eficaz e resolutivo. Este estudo contribuiu para ampliar o conhecimento neste temário ainda pouco explorado no Brasil ao evidenciar a necessidade de compartilhamento de experiências exitosas para o aprimoramento do gerenciamento de listas de espera.

\section{AGRADECIMENTOS}

Agradecemos a professora Melissa Moura Mello por ter realizado a revisão linguística-textual, gramatical e ortográfica de português deste artigo.

\section{REFERÊNCIAS}

1. AGUIAR LOF, LIRA ACO. Transparência no Sistema Único de Saúde - inciativas e desafios na divulgação eletrônica das listas de espera. Caderno Ibero-Americanos de Direito Sanitário, 2018; 7(2): 1-14.

2. ALBRECHT CAM, et al. O conceito de equidade na produção científica em saúde: uma revisão. Saúde soc., 2017; 26(1): 115-128. 
3. CLARKE J, et al. New geographic model of care to manage the post-COVID-19 elective surgery aftershock in England: a retrospective observational study. Rev BMJ Open, 2020; 10(10): 1-9.

4. FARIAS CML, et al. Tempo de espera e absenteísmo na atenção especializada: um desafio para os sistemas universais de saúde. Saúde em Debate, 2019; 43(5): 190-204.

5. GANGSTOE JJ, et al. Norwegian Priority Setting in Practice - an Analysis of Waiting Time Patterns Across Medical Disciplines. Rev Int J Health Policy Manag., 2016; 5(6): 373-378.

6. GIAMBERARDINO FILHO D. A falta de médicos e a atual situação do SUS. Audiência Pública. Brasília: Comissão Permanente de Direitos Humanos do Senado Federal, 2019. Disponível em: http://webcache.googleusercontent.com/search?q=cache:_2iWGMLa0I0J:legis.senado.leg.br/sdleggetter/documento/download/65a9c29d-32e0-4ee3-bb30-ddbae89835e4+\&cd=5\&hl=pt-BR\&ct=clnk\&gl=br. Acessado em: 15 de jun. de 2021.

7. JULIO C, et al. Waiting lists management model based on timeliness and justice. Rev Med Chil., 2016; 144(6): 781787.

8. LABERGE M, et al. Clinical pathway efficiency for elective joint replacement surgeries: a case study. Rev $\mathrm{J}$ Health Organ Manag., 2019; 33(3): 323-338.

9. LIPPI MC, et al. Waiting list management as an approach to planning and coordination of elective health services. Rev Gestão \& Saúde, 2018; 9(1): 159-177.

10. LISBÔA RL, et al. Actions of a regulatory nurse in the management of surgical waiting lists. Rev Bras Enferm., 2022; 75(2): e20201233.

11. LISBÔA RL, et al. Atuação do enfermeiro regulador de leitos na pandemia pela covid-19: relato de experiência profissional. Saúde Coletiva (Barueri), 2021; 11(70): 8812-8824.

12. LOGVINOV II, et al. Patient Survey of Referral From One Surgeon to Another to Reduce Maximum Waiting Time for Elective Surgery and Hours of Overutilized Operating Room Time. Rev Anesth Analg., 2018; 126(4): 1249-1256.

13. LUNGU DA, et al. Decision making tools for managing waiting times and treatment rates in elective surgery. Rev BMC Health Serv Res., 2019; 19(1): 369-377.

14. MELNYK BM, FINEOUT-OVERHOLT E. Evidence-based practice in nursing \& healthcare: a guide to best practice. 2. ed. Philadelphia: Wolters Kluwer/Lippincott Williams \& Wilkins, 2011.

15. MENDES KDS, et al. Integrative literature review: a research method to incorporate evidence in health care and nursing. Texto contexto-enferm., 2008; 17(4): 758-764.

16. MINISTÉRIO DA SAÚDE. Secretaria de Ciência, Tecnologia e Insumos Estratégicos. Departamento de Ciência e Tecnologia. Diretrizes Metodológicas Elaboração de revisão sistemática e metanálise de estudos observacionais comparativos sobre fatores de risco e prognóstico. 2014. Disponível em: https://bvsms.saude.gov.br/bvs/publicacoes/diretrizes_metodologicas_fatores_risco_prognostico.pdf. Acessado em 15 de jun. de 2021.

17. MOHER D, et al. Preferred Reporting Items for Systematic Reviews and Meta-Analyses: The PRISMA Statement. The PRISMA Group, 2009; 6(7): e1000097.

18. OLIVEIRA IS, et al. Desenvolvimento de software para gerenciamento de leitos na urgência e emergência. Rev Bras Enferm., 2021; 74(Suppl 5): e20200055.

19. OUZZANI M, et al. Rayyan - a web and mobile app for systematic reviews. Systematic Reviews, 2016; 5(1): $210-216$.

20. PAGE MJ, et al. The PRISMA 2020 statement: an update guideline for reporting systematic review. BMJ., 2021; 372(71): 01-09.

21. POMEY MP, et al. Wait time management strategies for total joint replacement surgery: sustainability and unintended consequences. Rev BMC Health Serv Res., 2017;7(1): 629-645.

22. RAY S, KIRTANIA J. Waiting time of inpatients before elective surgical procedures at a State Government Teaching Hospital in India. Rev Indian J Public Health, 2017;61(4): 284-289.

23. REGIS TKO, et al. Implementação do Lean Healthcare: experiências e lições aprendidas em hospitais brasileiros. Revista de Administração de Empresas, 2018; 58(1): 30-43.

24. REIGOTA SM, et al. The INTERMED method for patients with mental disorders: integrative literature review. Rev. USP., 2019; 52(2): 136-143.

25. SAGGAF MM, ANASTAKIS DJ. The impacto f COVID-19 on the surgical wait times for plastic and reconstructive surgery in Ontario. Plastic Surgery, 2021; 1(1): 1-12.

26. SANTOS LM, et al. Aplicabilidade da metodologia enxuta na organização de serviços de saúde: uma revisão integrativa. Rev Pesquisa, Sociedade e Desenvolvimento, 2020; 9(7): e345974054.

27. SANTOS MC, BALSANELLI APB. A implementação do lean healthcare em serviços de saúde hospitalares. Rev. Enferm UFPE., 2021;15: e247422.

28. SENNA SBB, et al. Gestão da fila de espera para cirurgias eletivas em hospitais do Sistema Único de Saúde. Brazilian Journal of Surgery and Clinical Research, 2020; 30(2): 79-82.

29. SICILIANI L, HURST J. Tackling excessive waiting times for elective surgery: a comparative analysis of policies in 12 OECD countries. Health Policy, 2005; 72(2): 201-215.

30. SILVA TO, et al. Gestão hospitalar e gerenciamento em enfermagem à luz da filosofia lean healthcare. Cogitare enferm., 2019; 24: e60003.

31. SILVA LID, et al. Avaliação do conhecimento dos acadêmicos de enfermagem em relação à Metodologia Lean: estudo transversal. REAS., 2021;13(4): 1-11.

32. VALSANGKAR NP, et al. Effect of Lean Processes on Surgical Wait Times and Efficiency in a Tertiary Care Veterans Affairs Medical Center. Rev JAMA Surg., 2017;152(1): 42-47. 